\title{
LE POINT DE VUE DE L'INDIGÈNE ou COMMENT ON ÉCRIT L'HISTOIRE DE LA LITTÉRATURE
}

$\ll \prod$ he final goal of which an ethnographer should never lose sight [...] realize his vision of his world ${ }^{1}$." C'est ainsi que, dans un passage célèbre de l'introduction aux Argonautes du Pacifique occidental, Bronislaw Malinowski définit le travail de l'ethnographe. L'analogie est tentante : en nous attachant à l'histoire de la littérature telle qu'elle est racontée par les écrivains eux-mêmes, nous procédons en somme comme des ethnographes soucieux de reconstituer la vision du monde des indigènes-leur rapport à la vie, leur vision de leur monde et donc aussi, leur rapport au passé. Venus du monde savant, du pays de l'université et des bibliothèques, nous cherchons à comprendre la façon dont ces étranges autochtones, les écrivains, racontent leur histoire et celle de ce pays de Littérature qu'ils habitent.

Quelle est leur relation au temps ? A la chronologie ? Quels sont leurs ancêtres réels ? Quels sont leurs ancêtres mythiques ? Comment s'inscriventils dans cette histoire qu'ils racontent ? Quel est le but avoué de leurs récits ? Quel est le but caché de leurs récits ?

Ces questions sont d'autant plus pertinentes que, au XX' siècle, la littérature est conçue comme une activité historique et bien souvent présentée comme telle. Faire de la littérature, c'est se situer dans le temps, avoir un passé et des prédécesseurs, pour s'affilier ou pour rompre avec eux, mais toujours en s'inscrivant dans un temporalité orientée. Pas seulement donc la mémoire, les souvenirs de l'école ou des premières lectures, mais bien l'histoire.

De sorte que faire de la littérature, c'est peut-être espérer faire l'histoire, mais c'est en tout cas toujours faire $d e$ l'histoire. A ce titre, cette tribu-les écrivainsque nous étudions est une société chaude au sens strict : nos indigènes ont une

1. Bronislaw Malinowski, Argonauts of the Western Pacific: an Account of Native Enterprise and Adventure in the Archipelagoes of Melanesian New Guinea [1922], Read Books, 2007, p. 25. (" Le but dernier que l'ethnographe ne doit jamais perdre de vue $[\ldots]$ consiste, en un mot, à saisir le point de vue de l'indigène, son rapport à la vie, sa vision de son monde. ")

The Romanic Review Volume 100 Numbers 1-2 @ The Trustees of Columbia University 
conscience historique ou, plus exactement, l'histoire est au nombre des catégories et des outils intellectuels qu'ils mobilisent pour se penser eux-mêmes ${ }^{2}$.

C'est ce qui fait que l'histoire littéraire des écrivains est intéressante : ce n'est pas seulement une autre version des faits, mais - en tout cas dès la fin du XIX siècle-, une véritable conscience de soi. L'histoire littéraire des écrivains, ce n'est pas la même histoire d'un autre point de vue, et ce n'est pas seulement un registre parmi d'autres de l'activité littéraire (qu'on pourrait ou non opposer à l'histoire littéraire savante) ; c'est un mode de pensée consubstantiel à l'activité littéraire et créatrice, qui l'imprègne jusque dans ses recoins : lorsque Rimbaud place un pronom non tonique à la césure d'un alexandrin, c'est un geste habité d'une conscience historique. Ce n'est pas que cela, mais c'est aussi cela. Le réductionnisme commence lorsqu'un tel geste est rabattu sur son intention historique-qu'il s'agisse de faire l'éloge de sa " modernité " ou au contraire de n'y voir qu'une habile tactique pour " prendre place dans l'histoire »-, de telles lectures étant tout aussi pauvres que celles qui voient dans un je à la césure l'effet d'une vitalité créatrice débordante qui n'aurait que faire des conventions.

Une question épineuse apparaît ici, et qui restera en suspens : celle de l'émergence historique de cette conscience historique. De la même façon qu'il n'y a pas de sociétés sans histoire, il n'existe pas de littérature soustraite au contexte et indifférente à son propre passé. Mais de la même façon que les hommes peuvent choisir ou non de penser l'histoire comme une contingence dont il faut se prémunir ou comme une force directrice qu'il faut accompagner et orienter, la manière dont les écrivains se rapportent au flux historique a elle-même historiquement varié. Y avait-il pour Racine des événements littéraires, y avait-il même une histoire littéraire? Au sens étroit, sans aucune doute-mais au sens où certaines œuvres auraient dessiné un avant et un après, auraient modifié la perception du passé et les conditions de l'exercice futur de la littérature ${ }^{3}$ ?

2. Voir Georges Charbonnier, Entretiens avec Claude Lévi-Strauss, Plon / Julliard, 1961, p. 46-48.

3. Revenant sur Le Degré zéro de l'écriture à la fin des Mythologies, Barthes situe vers 1850 cette " crise morale du langage littéraire ", au cours de laquelle l'être historique de la Littérature serait soudain apparue à la " conscience de l'écrivain " : "rejetant la fausse nature du langage, l'écrivain s'est violemment déporté vers une antinature du langage. " (Euvres complètes, t. 1, Paris : Seuil, 2002, p. 846-847). Dans " Pourquoi la littérature respire mal " (1960), Julien Gracq conçoit ce changement moins en termes de prise de conscience que de disjonction entre deux voies : à partir des années 1830-1840, explique-t-il, deux lignes se séparent et commencent à coexister : une " littérature de tradition et de continuité " et une " littérature de rupture ", celle des " fondateurs d'ordre », " pour lesquels l'avènement d'une esthétique signifie [...] par essence la mort de toutes les autres " (Euvres complètes, t. I, Paris : Gallimard, "Bibliothèque de la Pléiade», 1989, p. 861-863). 
Quoi qu'il en soit, dès que l'on s'intéresse à l'histoire littéraire des écrivains, dès qu'on l'a identifiée comme un objet possible d'enquête, on s'aperçoit qu'elle est, aux $\mathrm{XIX}^{\mathrm{e}}$ et $\mathrm{XX}^{\mathrm{e}}$ siècles, partout : dans les manifestes, les correspondances et les entretiens, bien sûr, mais aussi dans les constructions romanesques, l'architecture des recueils de poèmes et également dans quantités de textes et essais qui se prêtent mal à une distinction entre littérature primaire et secondarité critique.

Pendant trois ans, le programme de recherche international intitulé « L'histoire des écrivains ", entrepris à l'initiative de l'équipe "Littératures françaises du $\mathrm{XX}^{\mathrm{e}}$ siècle " de l'Université Paris-Sorbonne, a essayé de repérer et décrire les lieux et les façons dont cette histoire se fait. Il est d'abord apparu que l'histoire littéraire des écrivains est à l'œuvre dans des pratiques collectives : les collections, les anthologies, les groupes, les revues, etc ${ }^{4}$. Ensuite qu'elle a des outils conceptuels de prédilection : la génération, par exemple, notion caractéristique d'une histoire perçue de l'intérieur, pour laquelle la compréhension du passé est inséparable d'une mémoire et d'un rapport de filiation, avoué ou dénié. Et surtout au cours de ces colloques et journées d'études, est apparue non pas une pensée sauvage, mais une histoire sauvage, ou une façon sauvage de faire de l'histoire, délibérément différente dans son fonctionnement et ses principes de l'histoire savante ou domestiquée ${ }^{5}$ : cette histoire est instable, incohérente, discontinue ; elle peut occasionnellement s'ouvrir à la fiction ou se mêler de fiction ${ }^{6}$; elle est relativement indifférente à la chronologie et se prête volontiers aux télescopages, aux synchronies bizarres, aux déplacements et aux rapprochements inattendus ${ }^{7}$. Cette histoire " rechargée par un pro-

4. Voir, sur le site www.fabula.org/hle, les actes du colloque du 16 juin 2006 " Collections d'écrivains (1945-1980) " ainsi que, à paraître, Les Anthologies poétiques d'écrivains (1850-2000), Bern : Peter Lang, 2009.

5. Comme le fait remarquer Marielle Macé, la " constitution de l'histoire littéraire comme discipline et son passage dans l'enseignement » a conduit les écrivains du début du XX' siècle à entrer le plus souvent dans un rapport " de concurrence déclarée avec l'histoire universitaire, les manuels et les ouvrages savants-présentation pédagogique et recherche étant bien souvent confondus sans plus de nuance: les écrivains donnent le sentiment de précéder, anticiper, reconfigurer, améliorer ou récrire une histoire de la littérature supposée officielle et extérieure. [...] C'est pourquoi l'histoire littéraire des écrivains suppose le plus souvent, à l'état implicite, une histoire première exagérément unifiée, à refaire, à défaire, à déplacer, à prendre de vitesse ou encore à émietter puisque les histoires d'écrivains ne sont pas agençables entre elles, et atomisent le geste historien au profit de figures existentielles. " ("L'histoire littéraire à contretemps ", sur le site www.fabula.org/hle).

6. Voir, à paraître, "Fictions d'histoire littéraire ", La Licorne, $\mathrm{n}^{\circ} 86$, sous la dir. de Jean-Louis Jeannelle, Rennes : Presses universitaires de Rennes, 2009.

7. Voir les actes du colloque "Déplacements, dégagements" du 2 décembre 2005 sur le site www.fabula.org/hle ainsi que Bruno Curatolo (sous la direction de), Les 
gramme personnel », selon les mots de Marielle Macé, permet aux écrivains de " déployer la singularité du temps littéraire, [de] représenter le devenir de la littérature dans la littérature, [et d'] affirmer de diverses manières, par le goût de la rupture ou celui des rassemblements, que la force des œuvres est dans leur capacité à produire du temps, à en dévier le cours ". Ainsi se dessinent diverses configurations du temps des Lettres fondées sur des « télescopages insistants entre le présent et le passé et [la] pluralisation du présent lui-même ":

... la synchronie, les filiations, les couplages la rétrospection, la péremption [...], les rythmes du vieillissement, la mélancolie, l'inactualité, l'occupation du présent, la projection, l'attente, le guet, le délai . . . catégories historiographiques fermes ou floues, qui souvent « faussent » l'organisation de l'histoire savante parce qu'elles sont à la fois cognitives et existentielles et remplissent plusieurs fonctions, pour une communauté et pour un individu ${ }^{8}$.

Bien entendu, comme tout récit pro domo, les histoires littéraires d'écrivains sont marquées du sceau de la partialité, voire de la revendication ; elles fonctionnent souvent sur le modèle de la prophétie créatrice ou du wishful thinking. Elles sont orientées et déterminées autant par des rivalités présentes que par un pari sur l'avenir, et il importe pour l'écrivain-historien de bien choisir son ancêtre et de ne pas se tromper de lignée. Mais elles restent des histoires au sens où, comme la pensée sauvage, elles introduisent de l'ordre, elles ordonnent et configurent le passé.

$$
* * *
$$

Il est donc tentant d'opposer, dans un premier temps de l'analyse au moins, l'histoire littéraire savante à l'histoire littéraire des écrivains (ou aux histoires littéraires d'écrivains) comme la pensée scientifique à la pensée sauvage (ou aux formes diverses prises par la pensée mythique). On aurait là deux types d'entreprises séparées par des traits distinctifs : la première serait collective et relativement unifiée, la seconde serait éclatée, constituée d'un ensemble hétérogène d'histoires individuelles; la première serait désintéressée et tendrait à l'objectivité, les secondes seraient intéressées et subjectives, orientées par le souci de l'écrivain de s'inscrire à la pointe du récit qu'il produit ; la première serait une entreprise de connaissance constituant à elle-même sa propre fin quand les secondes auraient valeur de "défense et illustration " du projet littéraire qu'elles servent : La crise du roman : des lendemains du naturalisme

Ecrivains auteurs de l'bistoire littéraire, Besançon : Presses universitaires de FrancheComté, 2007.

8. Marielle Macé, «L'histoire littéraire à contretemps ", art. cit. 
aux années vingt est un livre qui se suffit à lui-même quand les généalogies surréalistes du Manifeste visent à configurer le paysage dans lequel le surréa lisme doit s'inscrire et surtout être lu. Au fond, c'est un peu la perspective des études de sociologie littéraire inspirées des Règle de l'art de Pierre Bourdieu : le sociologue se donne pour tâche la reconstitution de l'histoire objective du champ littéraire, et les écrivains, tous historiens eux aussi, se répartissent et se rangent selon la perspicacité de leur lecture et la justesse de leur perception de la dynamique du champ. Cette perspicacité est certes inconsciente et affaire d'habitus, c'est-à-dire de disposition incorporée à lire et " jouer le jeu ", mais il demeure frappant, lorsqu'on lit les travaux des sociologues "bourdieusiens », de voir que les écrivains « victorieux » dans la lutte pour l'acquisition du capital spécifique finissent toujours par apparaître comme des sociologues qui s'ignorent et qui agissent spontanément en conformité avec leur intérêt, quoique sans en passer par un décryptage conscient des logiques historiques. On aurait ainsi non plus deux types de lectures historiques (la savante et la sauvage), mais (au moins) trois : celle du sociologue savant, spécialiste de l'ingénierie du champ ; la lecture-sauvage et inconsciente d'elle-même, mais terriblement efficace-de l'écrivain perspicace et victorieux ; et toutes les lectures erronées des vaincus de l'histoire.

Pourtant, ce grand partage entre histoire savante et histoires sauvages n'est pas entièrement satisfaisant. D'abord en raison de la question du critère : quel est le critère final de distinction entre pensée scientifique et pensée sauvage ? Comme on le sait, il ne s'agit nullement de la pureté intellectuelle, de la rationalité ou de l'objectivité. Le sauvage « bricoleur " n'est pas englué dans la pratique et l'affectivité ; ses classifications relèvent d'un dessein proprement intellectuel, et elles visent la connaissance et l'exploitation du monde sensible, tout autant que les entreprises de l'ingénieur. En revanche, ses matériaux diffèrent, et ce à trois égards : au contraire de l'ingénieur, il opère sur un ensemble clos-son travail est donc toujours de réorganisation, et non d'ouverture d'un ensemble asservi à des fins définies ex abstracto-; les éléments qu'il emploie sont déjà " précontraints ", à demi particularisés, et leurs possibilités de réutilisation, quoique très nombreuses, ne sont pas infinies ; enfin, son travail est caractérisée par une homogénéité des moyens et des objets ${ }^{9}$, quand celui de l'ingénieur est caractérisé par une hétérogénéité des moyens et des objets puisqu'il crée ses propres instruments et opère au moyen de concepts sur un ensemble naturel, ce dernier étant en théorie indéterminé et virtuellement infini (même si dans les faits, il ne l'est jamais complètement). La différence

9. La pensée sauvage vise "l'organisation et [. . .] l'exploitation spéculatives du monde sensible en termes de sensible " : il s'agit toujours de connaître, au moyen de signes, un monde lui-même envisagé comme un ensemble de signes, à mi-chemin du percept et du concept (Claude Lévi-Strauss, La Pensée sauvage [1962], in CEuvres, Paris : Gallimard, "Bibliothèque de la Pléiade ", 2008, p. 576). 
de rendement pratique est la conséquence de cette série de contrastes entre ces deux modes de pensée «également valides ${ }^{10}$.

Mais ces contrastes s'appliquent mal à la différence supposée entre l'histoire littéraire savante et l'histoire littéraire sauvage des écrivains. L'une et l'autre travaillent sur le même ensemble, qui est vaste mais clos : l'une comme l'autre sont, comme la pensée mythique, "prisonnières d'événements et d'expériences qu'elles disposent et redisposent inlassablement pour leur découvrir un sens ${ }^{11}$. Il est par ailleurs évident que les " objets " auxquelles elles s'appliquent sont les mêmes, et sont fortement précontraints, puisqu'il s'agit non d'éléments du monde sensibles réduits à leurs qualités premières et ouverts à tous les usages possibles, mais de textes et d'œuvres, c'est-à-dire d'unités textes-auteurs. C'est à propos des instruments que la différence semble la plus nette. Elle n'est pourtant pas radicale. On peut bien sûr comparer les vertus heuristiques des concepts, et opposer le champ à la génération comme un concept plus rigoureux à un concept moins rigoureux ${ }^{12}$ ou, plus justement, comme des concepts plus ou moins transparents à la réalité à laquelle ils s'appliquent : un instrument comme celui de génération n'est pas une abstraction pure, créée pour les besoins de la cause; il est encore " pris " dans la signification en quelque sorte et il conserve quelque chose de la réalité dont il traite ; comme le signe, il « accepte, et même exige qu'une certaine épaisseur d'humanité soit incorporée à cette réalité ${ }^{13}$. Pourtant, la génération n'est pas comme l'écureuil ou la sauge dans un mythe nord-américain ; c'est déjà une abstraction qui a rompu ses attaches avec la perception, et on ne peut tout à fait lui dénier le statut de concept. On savait déjà que la pensée scientifique et la pensée mythique ne s'opposaient pas comme l'objectivité à la subjectivité ni comme le savoir désintéressé à la connaissance intéressée ; on voit à présent que la distinction entre histoire littéraire universitaire et histoire littéraire indigène est bien plus ténue encore, non pas parce que la seconde est, elle aussi, savante, mais au contraire parce que la première est elle-même très bricoleuse.

\section{$x * x$}

Il faut ensuite ajouter un élément, à propos de cet univers de textes auquel les historiens s'appliquent. Comme on l'a dit, à l'instar du "trésor " du bricoleur, cet univers est clos et le procès de connaissance consiste d'abord en un

10. Ibid., p. 582 .

11. Ibid.

12. Voir par exemple la réflexion de Pierre Nora: «... les historiens les plus responsables, tout en sentant l'irremplaçable lumière que permettait de jeter la génération sur l'intelligence des temps, [ont] globalement récusé le concept comme schématique, inefficace, grossier, et en définitive moins enrichissant que réducteur. " ( La génération ", Les Lieux de mémoire, t. II [1986], Paris : Gallimard, "Quarto », 1997, p. 2983.)

13. La Pensée sauvage, op. cit., p. 580. 
travail de réorganisation et d'arrangement. Mais surtout, cet univers n'est pas constitué d'éléments objectifs mais de valeurs. L'idée que l'historien savant se présente face aux faits et les ordonne rationnellement est déjà, on le sait, une fiction, mais que dire alors de l'historien de la littérature ?

Il ne s'agit pas ici d'adopter la position de Max Weber définissant toute histoire comme un travail de valorisation (puisqu'on opère un tri et sélectionne certains traits du passé aux dépens d'autres au nom d'un idéal implicite et in fine injustifiable), mais de reconnaitre que les objets dont s'empare l'historien de la littérature, qu'il soit écrivain ou universitaire, sont déjà valorisés et se distinguent sur un vaste fond de textes non-remarquables, autrement dit que ces objets sont perçus comme singuliers et envisagés comme tels. Or voilà précisément ce que ne font pas les historiens " purs ", qui ne s'intéressent jamais à l'individualité que pour la comprendre et retrouver en elle une forme de généralité. Comme l'écrit Paul Veyne, "L'histoire commence [toujours] par une dévalorisation générale » : « Brigitte Bardot et Pompidou ne sont plus des individualités notoires, admirées ou désirées, mais les représentants de leur catégorie ; la première est une star, la seconde se partage entre l'espèce des professeurs qui se tournent vers la politique et l'espèce des chefs d'Etat. On est passé de la singularité individuelle à la spécificité, c'est-à-dire à l'individu comme intelligible (c'est pourquoi "spécifique" veut dire à la fois "général" et "particulier") ${ }^{14}$. "L'historien de la littérature ne s'intéresse pas à la catégorie des recueils de poèmes publiés en 1857, mais aux Fleurs du mal dans leur singularité admirable, et s'il s'intéresse à la première, c'est évidemment pour mieux saisir les secondes : Les Fleurs $d u$ mal n'ont pas pour lui valeur d'échantillon.

C'est aussi une des limites de l'analogie avec l'ethnographie. Les ethnographes ont pour principe de ne pas distinguer entre le haut et le bas de la culture. Ils s'attachent tout autant aux ordures et aux techniques du corps qu'aux productions culturelles élevées. Or au principe de l'histoire littéraire, il y a un refus de ce nivellement; nous ne nous intéressons pas à tous les textes, mais donnons priorité à ceux que nous aimons et qui nous importent, c'est-à-dire aussi à ceux qui, au moment où nous écrivons, ont gagné cette bataille pour la postérité.

Veut-on dire par là que les historiens de la littérature ressemblent fatalement à ces adeptes de la "critique interne " méchamment décrits par Bourdieu, amateurs mystifiés et idéalistes, dont le discours ne vise qu'à sans cesse confirmer la grandeur d'une ouvre aveuglément sacralisée ${ }^{15}$ ? Certes, non.

14. Paul Veyne, Comment on écrit l'bistoire [1971], Paris : Le Seuil, «Points ", 1996, p. 81.

15. Voir, dans Les Règles de l'art, les pages consacrées à la "doxa littéraire " et à " la vénération de tous ceux qui ont été dressés, souvent dès leur prime jeunesse, à accomplir les rites sacramentels de la dévotion culturelle ". (Paris : Le Seuil, "Libre examen ", 1992, p. 259). 
On sait bien que les historiens de la littérature envisagent-et souvent avec talent, et minutie, et dévouement-l'ensemble des recueils publiés en 1857 (ou l'évolution du sonnet, ou les poètes orphelins de père, ou tout autre catégorie ou intrigue dont Les Fleurs du mal peuvent relever à un titre ou un autre), mais ils ne font jamais uniquement cela et reviennent toujours aux Fleurs du mal. De deux choses l'une : ou l'histoire de la littérature est une véritable histoire, et dans ce cas, elle est une histoire du livre, une histoire de la lecture ou une histoire du goût qui trouvera dans les œuvres des illustrations et des confirmations; ou elle prend pour objet la littérature comme ensemble de singularités valorisées (c'est-à-dire valorisées aujourd'hui) et dans ce cas, elle n'est pas une histoire.

Bien entendu, l'histoire de la littérature telle qu'elle est effectivement pratiquée se situe toujours entre les deux, et ne cesse d'aller d'une position à l'autre : tantôt histoire sérieuse, appuyée sur des sources, envisageant le contexte, créant des catégories, multipliant les intrigues dont relève l'œuvre envisagée ; tantôt revenant au texte et utilisant cette histoire "pure " pour confirmer, par un jeu de contrastes et de différences, la valeur et la singularité de l'œuvre. Ce qu'on appelle " histoire de la littérature " relève donc de cette catégorie hybride que Paul Veyne appelle les « histoires axiologiques ", c'està-dire en fait d'un discours qui passe sans cesse de l'histoire pure à l'axiologie et vice-versa : " l'historien axiologiste parlera de la vie littéraire sous Louis XIV pour expliquer la vie et l'œuvre de Racine, il ne verra pas en Racine un figurant de cette vie littéraire comme ferait l'historien pur. » Cela ne veut pas dire que l'histoire pure, quand elle s'applique à la littérature, ne comporte pas des jugements de valeur, mais ceux-ci sont « au discours indirect, autrement dit, à l'état de jugements de fait " ${ }^{16}$. Toute bonne histoire littéraire commence par envisager l'œuvre « en son temps » et en effet, il importe de savoir ce que les contemporains ont pensé des Fleurs du mal et pourquoi ils ont aimé le recueil ou l'ont condamné. Mais on sait assez que ces évaluations ne sont plus les nôtres et qu'elles font donc partie de ces faits que l'on mobilisera au service de la singularisation et de la valorisation du recueil. C'est ce qui fait, d'ailleurs, que l'historien de la littérature n'est pas lui-même, contrairement à ce qu'on dit parfois, d'abord en position d'évaluation ; à moins de se pencher sur une littérature extrêmement contemporaine, encore soustraite à toute évaluation (ce qui est un cas idéal et à peu près impossible car les textes ne surgissent pas

16. Comment on écrit l'histoire, op. cit., p. 97. Sur l'hétérogénéité constitutive de l'histoire littéraire, voir aussi Jean-Louis Jeannelle, "Pré-histoires littéraires : qu'est-ce que l'histoire littéraire des écrivains? " in Bruno Curatolo (sous la direction de), Les Ecrivains auteurs de l'histoire littéraire, op. cit., p. 21-25. 
du néant comme des aérolithes), il hérite toujours d'une évaluation qu'il peut confirmer ou infirmer mais que, le plus souvent, il lui suffit de suivre ${ }^{17}$.

On voit poindre ici la question de la circularité de l'histoire littéraire. On sait que Thibaudet compare souvent la littérature à un paysage, et l'analogie fonctionne bien ici : dans ses enquêtes, l'historien arrive dans un pays doté de reliefs, et il ne dépend pas de lui de le modifier. Nous nous attachons en priorité aux œuvres que l'histoire nous a léguées, c'est-à-dire aux histoires littéraires d'écrivains victorieuses, victoire que par une circularité inhérente à notre profession, nous confirmons et fortifions par nos études. Car n'oublions pas que les écrivains sont pour ainsi dire nos seules sources : nous les étudions, nous les situons, mais toujours à partir de ce qu'ils nous disent ${ }^{18}$. Et dans cette bataille, il y a des perdants : qui se souvient de l'histoire littéraire d'Eugène Montfort qui, en 1925, prétendait qu'à la pointe de la littérature française du tournant du XXe siècle, n'était nullement André Gide et Stéphane Mallarmé mais Paul Bourget, Jean-Louis Vaudoyer et Abel Hermant ${ }^{19}$ ?

Il reste que cette circularité, si elle est une fatalité pour l'historien de la littérature, ne doit pas pour autant devenir régulatrice. L'historien doit sans doute en prendre conscience, la reconnaître, admettre aussi qu'il ne peut à lui seul abattre les montagnes et rehausser les minores, mais il ne doit pas pour autant considérer ce paysage et ses reliefs comme un donné. Ce serait retomber, par l'autre bout en quelque sorte, dans les erreurs de l'histoire positiviste. Pendant longtemps en effet, cette circularité est demeurée inaperçue, ou réprimée. Pour éviter les dilemmes qui l'accompagnent, on disposait d'un instrument très efficace, qui fonctionnait comme un véritable alibi : la notion d'épreuve du temps. Les œuvres arrivées jusqu'à nous avaient passé l'épreuve du temps, c'était un signe objectif de leur valeur-et si d'aventure on doutait de cette valeur, il suffisait d'invoquer la survie des ouvres pour la confirmer. La permanence confirmait la valeur ; et la valeur justifiait la permanence ${ }^{20}$.

17. D'où ce paradoxe fort explicable dont parle Paul Veyne, qui autorise l'historien de la littérature à " avoir le goût mauvais " : "Il suffira, pour être bon historien axiologique, qu'il emprunte à la voix publique la liste canonique des grands écrivains ; après quoi, il saura qu'il lui faut analyser la vie et l'œuvre de Baudelaire plutôt que de Béranger. " (ibid., p. 98)

18. Voir sur ce point la réflexion très éclairante de Jean-Louis Jeannelle : « Pré-histoires littéraires : qu'est-ce que l'histoire littéraire des écrivains ? ", art. cit., p. 21-30.

19. Voir Eugène Montfort (sous la direction de), Vingt-cinq ans de littérature française, tableau de la vie littéraire de 1895 à 1920, Librairie de France, s.d. (1924-1927), et en particulier le chapitre «L'évolution de la langue et du style», par Pierre Lièvre. Voir aussi, sur le site www.fabula.org/hle la page consacrée à cet ouvrage.

20. Voir sur ce point, Judith Schlanger, La Mémoire des ceuvres, Paris : Nathan, « Le Texte à l'œuvre », 1992, p. 102-104 en particulier. 
Nous sommes aujourd'hui plus relativistes et connaissons trop les caprices du goût, les déterminations culturelles et les mécaniques sociales pour croire à une telle objectivité du canon. Mais ce scepticisme de bon aloi doit s'appliquer tout autant à nos sélections et jugements présents. D'abord parce que trop d'oublis, de disparitions inattendues en même temps que de redécouvertes enseignent la prudence en ce domaine. On se souvient du chapitre "Attardés et égarés " que, dans son Histoire de la littérature française parue en 1895, Lanson consacrait à L'Astrée, d'Aubigné, Saint-Amant et Mlle de Scudéry : ceux-ci n'y apparaissaient que comme exemples « des résistances, des reculs, des contradictions, des aberrations de toute nature ", sur la voie glorieuse qui conduisait de Malherbe à Boileau. Heureusement, ajoutait Lanson, « il n'était pas en la puissance du passé de barrer la route à l'avenir " ${ }^{21}$. Inutile de dire que notre perception de la littérature du XVII siècle a changé, et que celle-ci s'organise en un tout autre paysage pour un historien d'aujourd'hui.

Mais c'est surtout la nature de ce " paysage " qui doit inciter à la prudence. Encore une fois, les grandes œuvres et les minores qui en dessinent les contours, les premiers plans et les ombres portées qui en étagent la profondeur, ne sont pas des donnés offerts à l'intelligence de l'historien. Que le modernisme d'Apollinaire ait éclipsé l'unanimisme de Jules Romain n'est pas un fait historique dont il faut rendre raison, car ce n'est pas un fait ; ce qui est un fait historique est que les hommes d'aujourd'hui, se penchant sur la littérature du début du $\mathrm{XX}^{\mathrm{e}}$ siècle, retiennent davantage le modernisme que l'unanimisme. De l'étude de l'histoire littéraire des écrivains, on peut au moins retirer ce bénéfice : en replaçant l'historien savant "dans le temps instable des décisions et des risques ", " emporté vers l'éventuel "22, elle prémunit contre la réification des verdicts de l'histoire. Tel est au fond le travers essentiel de la sociologie critique du champ littéraire : informée-quoi qu'elle en ait-par le goût du jour dans le choix des textes auxquels elle s'attache, elle finit toujours par ériger le présent en norme. La définition de la littérature est l'enjeu de luttes symboliques entre différentes fractions du corps social, nous dit-on ; est littérature ce que la fraction la plus autonome du champ littéraire consacre comme telle. En vertu de cette « loi ", l'observateur peut dès lors analyser avec délectation la circulation du capital symbolique, les rapports de force, et montrer sans grand risque que, dès le début, tout concourait à ce qui n'est qu'en apparence un " état de fait " : notre appréciation présente de la littérature passée. Outre le risque de tautologie explicative (il y a eu bataille, il y a eu défaite, et la défaite est expliquée par l'arrivée des vainqueurs ${ }^{23}$ ), outre

21. Histoire de la littérature française [1895], Paris : Hachette, 1951, p. 366, p. 372. 22. J'emprunte ces deux formules à Michel Murat, qui les emploie ici même dans son article "L'histoire littéraire, sujet de roman ».

23. Voir Vincent Debaene, «Le lettré et la division sociale du travail. Inactualité d'Agathon ", sur le site www.fabula.org/hle. 
que l'homologie postulée entre les positions des acteurs dans le champ et leurs choix esthétiques conduit souvent à négliger une part de la production ${ }^{24}$, la sociologie critique du champ littéraire ignore ainsi que même la littérature du passé est constamment l'objet de réévaluations et de nouveaux récits. Il n'est pas question bien sûr de nier le poids des jugements hérités en présentant I'histoire littéraire comme un récit en constante mutation, objet d'une réinvention continuelle, etc., ni de surestimer les capacités de l'historien à modifier la donne qui lui échoit ; il s'agit simplement de ne pas confondre les valeurs et les faits. On ne s'étonnera donc pas que, en dépit de sa neutralité affichée et de son relativisme de principe, cette sociologie reconduise toujours le point de vue des dominants ${ }^{25}$, c'est-à-dire, bien souvent à propos du $\mathrm{XX}^{\mathrm{e}}$ siècle, le métarécit moderniste des avant-gardes : le mouvement historique de la littérature est un mouvement d'essentialisation et de purification. Les ressorts explicatifs sont très différents puisque d'un côté on évoque une essence de la littérature et de l'autre la quête d'autonomie du pôle le plus avancé du champ, mais les conclusions sont curieusement identiques.

En est-on réduit à retirer tout privilège à l'histoire littéraire savante ? Bricoleuse comme l'histoire littéraire des écrivains, condamnée au va-et-vient entre les faits et les valeurs, souvent aveuglée par son finalisme et condamnée à « l'après-coup de la chose jugée », elle paraît n'avoir guère de mérites—sinon celui de l'érudition-et on voit mal ce qui la rend supérieure aux histoires littéraires d'écrivains. Faut-il conclure sur la triste et banale opposition entre les écrivains, créateurs pleins de sève et d'invention, même dans le regard qu'ils portent sur le passé, et les professeurs, toujours en retard d'un train, toujours

24. Ainsi Denis Hollier a-t-il relevé que le nom de Blanchot n'apparaissait quasiment pas dans La Guerre des écrivains de Gisèle Sapiro. Voir, sur ces questions, le compte rendu de ce livre par Laurent Jeanpierre : "Politiques de l'écrivain ou politiques de l'écriture ? ", French Politics, Culture \& Society, Vol. 19, No. 1, Spring 2001, p. 70-81.

25. Jean-Louis Jeannelle repère un semblable effet de confirmation dans La Guerre des écrivains, qu'il attribue de façon très convaincante à une forme de détermination par les sources : «On est frappé de constater que les analyses proprement sociologiques servent en grande partie à confirmer ou à infirmer une représentation globale de la période que Gisèle Sapiro a puisée dans les archives, les journaux ou les Mémoires littéraires de l'époque. La sociologue s'est en effet appuyée sur des graphiques fixant la position des écrivains dans le champ littéraire [...] mais in fine son étude s'est nourrie de quantités d'archives-correspondances, témoignages, souvenirs-qui constituaient autant d'ébauches narratives que Gisèle Sapiro s'est employée à évaluer et corriger. " ("Pré-histoires littéraires : qu'est-ce que l'histoire littéraire des écrivains ? ", art. cit., p. 28-29). 
du côté de l'institution et de la légitimation rétrospective ? Ou dans une veine moins mélancolique, sur un œcuménisme libéral donnant à toutes les histoires droit de cité sur un pied d'égalité ? Après tout, professeurs et écrivains n'habitent pas le même pays ; il est normal que chacun ait sa version des faits ; autres temps, autres mœurs ; autres lieux, autres histoires. C'est la solution du post-modernisme. Tout récit est par nature partiel, partial et surdéterminé à la fois par son contexte de production et son contexte de réception. Bien sûr, dira-t-on, les histoires d'écrivains ne sont pas neutres ; comme les histoires indigènes telles qu'elles sont livrées à l'ethnologue, elles sont orientées et déterminées par des luttes et des rivalités locales. Mais l'histoire littéraire savante n'est pas davantage libre de ces déterminations. Pourquoi s'obstiner à lui accorder un privilège que rien ne justifie ? A l'ethnologue classique, crispé sur ses procédures et ses modes d'écriture, l'ethnologue " post-moderne " objecte : "La traduction d'une forme culturelle étrangère ne trouve pas nécessairement sa forme la meilleure dans la monographie ethnographique et dans certaines conditions, il est possible qu'une performance dramatique, une danse ou un morceau de musique soient plus appropriés. "Un autre dit : "Des romans écrits par un Samoan peuvent parfaitement rivaliser avec le portrait de son peuple par un anthropologue distingué ${ }^{26}$. "Doit-on pareillement s'incliner devant les récits historiques des écrivains au prétexte qu'ils sont parcellaires, incomplets, intéressés, subjectifs, relatifs, etc.-et qu'ils sont tout cela pas plus, mais pas moins que les histoires savantes?

C'est ici sans doute qu'il faut tout concéder au post-modernisme, sauf son moralisme-car c'est ce dernier qui lui interdit la comparaison entre les récits (et non, comme on le dit, l'impossibilité de définir des critères objectifs d'évaluation). La question n'est pas de savoir si le récit de l'indigène «peut rivaliser " avec le récit de l'ethnologue (dans quel championnat ?), ni si l'histoire littéraire savante est scientifique (elle ne l'est pas, et elle l'est d'autant moins qu'aucune histoire ne l'est) mais plutôt de savoir si le savant sait mieux que les acteurs ce que ceux-ci font. Et la réponse est oui, pour autant qu'elle l'est toujours. Mais cela ne tient nullement à une grâce particulière, à une distribution initiale du savoir qui ferait que le savant aurait reçu en partage la rationalité ou la capacité spéculative quand l'indigène aurait reçu la pratique et l'inventivité. Ce savoir, cette capacité plus grande à écrire l'histoire d'un pays plus grand est un simple effet de position : si le pays est plus grand, c'est qu'il est vu de plus loin et de plus haut. Les hypothèses sur l'objectivité des uns, la subjectivité des autres, les tentatives de justification des " grands partages ",

26. Respectivement Talal Asad, "The Concept of Cultural Translation in British Social Anthropology ", in James Clifford and George Marcus (ed.), Writing Culture : the Poetics and Politics of Ethnography, Berkeley: University of California Press, 1986, p. 159 et James Clifford, « On Ethnographic Allegory », ibid., p. 119. 
les réflexions sur l'impossible neutralité de l'observateur ne sont pas seulement hautement spéculatives; elles sont, dans le cas de l'histoire littéraire, tout à fait inutiles. Il est évident que l'historien du surréalisme-s'il dispose de la capacité de sympathie suffisante-peut mieux faire l'histoire du surréalisme que les surréalistes eux-mêmes : il sait comment ceux-ci envisageaient leur propre généalogie ; il dispose à peu près de toutes les sources dont ils disposaient, de centaines d'informations qu'ils ne pouvaient pas connaître, et d'une capacité de mise en perspective (temporelle, spatiale, sociologique, etc.) que leur position leur interdisait. Et si cette mise en perspective leur était impossible, ce n'est pas parce que les surréalistes étaient englués dans la pratique ou aveuglés par leur intérêt et leur souci "d'entrer dans l'histoire ", mais simplement parce qu'il est inévitable que la conscience voie dans le passé " l'édification du présent ", c'est-à-dire considère le monde humain dans lequel elle évolue comme un terme, achevé par son action même. "A quelque époque que l'on se place, les hommes "primitifs" non moins que civilisés ont toujours su que leur destin serait en partie ce qu'ils le feraient être par leur action. Ils ont su aussi qu'il s'était écoulé du temps avant eux ; mais la connaissance de ce temps leur demeure étrangère, parce que l'action n'englobe pas la connaissance du passé et n'a pas non plus l'usage de cette connaissance ${ }^{27}$. " Pour échapper aux affres du relativisme et à l'insoluble concurrence des points de vue (vérité de l'ethnologue contre vérité de l'indigène), il suffit de renoncer à penser le rapport entre savant et non savant en termes de lucidité et de confondre connaissance et transparence à soi-même. Pas plus que les autres hommes, les écrivains ne savent l'histoire qu'ils font. La conscience réflexive que les hommes prennent d'eux-mêmes n'est pas un savoir ; pour l'ethnologue comme pour l'historien, ce n'est qu'un élément d'information supplémentaire.

Après avoir vu que l'histoire littéraire savante n'est pas tout à fait une histoire, il restait donc à admettre que l'histoire littéraire des écrivains n'en est presque pas une ; elle ne l'est qu'au sens où elle met en ordre le passé et le dispose en vue du présent, c'est-à-dire en vue d'une création présente. Cela ne lui ôte son intérêt en rien, bien au contraire, puisque comme le montrent les pages qui suivent, ces récits " historiques " jouent un rôle crucial dans le processus de création lui-même et bien souvent le conditionnent. Les histoires littéraires d'écrivains méritent sans aucun doute d'être examinées, mais moins au titre de contenus (éventuellement concurrents de l'histoire universitaire) qu'au titre de fonctions, non pas en tant que telles, donc, mais parce qu'elles aident à mieux percevoir ces singularités à quoi l'histoire littéraire revient toujours.

27. Paul Veyne, op. cit., p. 101-104. 
"Loin que la recherche de l'intelligibilité aboutisse à l'histoire comme à son point d'arrivée, c'est l'histoire qui sert de point de départ pour toute quête de l'intelligibilité ", écrivait Lévi-Strauss dans La Pensée sauvage, refusant de distinguer entre la raison du savant et celle de l'indigène en termes de conscience historique $^{28}$. Une telle formule est encore plus vraie de la littérature que des autres objets auxquels l'historien s'applique ; dans ce domaine moins encore qu'ailleurs, le récit historique ne peut constituer la fin dernière.

Columbia University

28. Claude Lévi-Strauss, La Pensée sauvage, op. cit., p. 841. 